\title{
The effects of parallel bars, body weight support and speed on the modulation of the locomotor pattern of spastic paretic gait. A preliminary communication
}

\author{
M Visintin MSc \& H Barbeau PhD
}

\begin{abstract}
School of Physical and Occupational Therapy, McGill University, Montreal, Quebec, Canada.
\end{abstract}

The effects of walking with and without parallel bars, providing $40 \%$ body weight support (BWS) and increasing speed on the gait pattern of spastic paretic subjects during treadmill locomotion were investigated. In asymmetrically involved subjects, walking without parallel bars led to a more symmetrical gait pattern with decreased compensation of the less involved side. This was accompanied by changes in electromyographic (EMG) and sagittal angular displacement profiles which favoured a more normal swing phase of the more involved limb. When symmetrically involved subjects walked without parallel bars, increases in EMG activity, with prolonged activation during the stance phase were noted, especially in the distal muscles. Providing $40 \%$ BWS facilitated gait when walking without parallel bars especially in the asymmetrically or severely involved subjects who showed marked difficulty at $0 \%$ BWS. Forty percent BWS led to a decrease in clonus associated with walking without parallel bars. Higher treadmill speeds increased clonus in some subjects while in others it only caused a small increase in EMG amplitude. Implications for gait training are discussed.

Keywords: spastic gait; parallel bars; body weight support; speed; electromyography; spinal cord injury.

\section{Introduction}

Incomplete spinal cord lesions in man commonly result in disturbances of the locomotor pattern. Altered recruitment patterns with premature muscle activation, as well as prolonged electromyographic (EMG) activity and delayed muscle relaxation have been identified in spastic paretic gait. ${ }^{1}$ The pattern may also be coupled with flattening of the EMG profiles, characterized by diminished or abolished peaks of EMG activity. Knutsson ${ }^{2}$ identified (1) early stretch activation of distal muscles, (2) paresis and (3) abnormal coactivation of agonist and antagonist lower limb muscles in response to loading, as likely to interfere with gait following a spinal cord lesion. Coupled with

Correspondence: Martha Visintin MSc, Jewish Rehabilitation Hospital, Physiotherapy Department, 3205 Place Alton Goldbloom, Chomedey, Laval, Quebec, Canada H7V 1R2. disturbances in locomotor programming, external factors such as the use of parallel bars, body weight support (BWS) and walking speed can also influence the locomotor pattern. Such external factors can be manipulated when retraining gait, aiming at optimizing the locomotor output.

Although widely used in the clinical setting, the use of parallel bars during gait training appears controversial. Conrad et $a l^{1,3}$ report that the decreased stability incurred during treadmill locomotion without parallel bars leads to a deterioration in the gait pattern of spastic paretic subjects. In contrast, conventional gait training for neurological patients discourages the use of parallel bars as it is believed to lead to an asymmetrical gait with compensation of the less involved side. ${ }^{4}$

The use of BWS, provided by an overhead harness which supports a percentage of body weight as subjects walk on a treadmill, has been proposed to retrain gait following a 
spinal cord lesion. BWS has been shown to facilitate gait and elicit a more normal gait pattern with respect to sagittal angular displacement patterns, temporal distance parameters, and EMG activity of lower limb muscles in a group of spastic paretic subjects during treadmill locomotion at their comfortable speed. ${ }^{5}$ Futhermore, with BWS, subjects were able to walk at higher comfortable treadmill speeds.

The characteristic slow walking speeds evident among spastic subjects ${ }^{5,6}$ is an additional concern when retraining gait. Gait training aims at increasing the walking speed, attempting to make the gait more efficient. However, few quantitative data exist to elucidate the effects of walking speed on a spastic paretic gait pattern.

It is evident that a better understanding of the effects of external factors such as parallel bars, BWS and speed on spastic paretic gait is required before a comprehensive gait training strategy can be developed. It is the aim of this study to quantitatively and qualitatively describe the effects of walking with and without parallel bars, with BWS, and at increased treadmill speeds on the locomotor pattern of spastic paretic subjects.

\section{Methods}

The study was conducted in the human gait laboratory which has previously been described in detail. ${ }^{7}$ Eight spastic paretic subjects, ranging in age from 22 to 42 years (mean $=27.5$ years), participated in this study. Seven subjects had sustained a traumatic incomplete spinal cord lesion to the cervical or thoracic spine, one suffered from nonfamilial progressive spastic paraparesis and one subject had a surgically induced lesion at the level of T10 following resection of a spinal tumour. Each subject was capable of independent overground locomotion with or without the use of external aids. The chronicity of the lesion ranged from 7 months to 21 years. The demographic data, presence of ankle clonus at rest, symmetrical involvement of the lower limbs, comfortable and maximal treadmill speed, and an overground ambulation profile for each subject are summarized in Table I.

\section{Body weight support}

The subjects walked on a treadmill while $0 \%$ BWS (full weight bearing) and $40 \%$ BWS were provided. The BWS apparatus consisted of a custom-designed harness which mechanically supported the patient vertically over the treadmill. The harness consisted of a pelvic band attached around the hips and two padded straps which pass between the legs to attach anteriorly to the pelvic band. The percentage of BWS provided was calibrated using a force transducer. The force was normalized to each subject's weight $(100 \%)$ and the sequence of $\%$ BWS provided $(0 \%$ or $40 \%)$ was randomly assigned into two trials given within the same experimental session. Prior to data collection, each subject was habituated at $0 \%$ BWS for 1-5 minutes according to his walking tolerance. During this practice session, treadmill speed was slowly increased from $0.0 \mathrm{~ms}^{-1}$ up to each subject's comfortable walking speed. It was also determined whether the subjects could walk at more than one treadmill speed (Table I). All subjects, except subject 2, were able to walk at a minimal and at a comfortable treadmill speed. Four subjects $(1,3,5,8)$ were able to walk at a maximal treadmill speed. Data were then collected for predetermined speeds (minimal, comfortable and maximal speeds) during subtrials at $0 \%$ BWS with parallel bars. Data were also collected at $0 \%$ and $40 \%$ BWS, without parallel bars at minimal or comfortable speeds (subjects: 1 , $2,3,6)$. Of the eight subjects, only one (8) required a short leg brace to control foot drop of the left ankle while walking on the treadmill.

A 10 minute rest period was given between each BWS trial to minimize fatigue. Blood pressure and pulse were monitored following each trial to control for undue stress on the subjects.

\section{EMG and footswitch data}

EMG activity was recorded from the gluteus maximus (GM), vastus lateralis (VL), medial hamstrings $(\mathrm{MH})$, tibialis anterior (TA), medial gastrocnemius (GA), and lateral soleus (SOL) of either the left (subjects 1, 4, 5, 6, 7) or right (subjects 2, 3, 
Table I Details of subjects

\begin{tabular}{|c|c|c|c|c|c|c|c|}
\hline $\begin{array}{l}\text { Subject } \\
\text { Sex } \\
\text { Age }\end{array}$ & $\begin{array}{l}\text { Level } \\
\text { of } \\
\text { lesion }\end{array}$ & $\begin{array}{l}\text { Chronicity } \\
\text { (years) }\end{array}$ & $\begin{array}{l}\text { Ankle } \\
\text { clonus }\end{array}$ & $\begin{array}{l}\text { Symmetrical } \\
\text { involvement }\end{array}$ & $\begin{array}{l}\text { Comfortable } \\
\text { treadmill } \\
\text { speed }\left(\mathrm{ms}^{-1}\right)\end{array}$ & $\begin{array}{c}\text { Maximal } \\
\text { treadmill } \\
\text { speed }\left(\mathrm{ms}^{-1}\right)\end{array}$ & $\begin{array}{l}\text { Overground walking } \\
\text { aids used }\end{array}$ \\
\hline $\begin{array}{l}1 \\
M \\
32\end{array}$ & SP & 3.0 & $\begin{array}{l}+(\mathrm{R}) \\
++(\mathrm{L})\end{array}$ & Yes & 0.40 & 0.60 & $\begin{array}{l}\text { Bilateral foot } \\
\text { drop braces }\end{array}$ \\
\hline $\begin{array}{r}2 \\
M \\
23\end{array}$ & C6-7 & 1.5 & $+++(\mathrm{Bil})$ & $(\mathrm{R}>\mathrm{L})$ & 0.08 & 0.08 & $\begin{array}{l}\text { (L) long leg brace; } \\
\text { Canadian crutches }\end{array}$ \\
\hline $\begin{array}{r}4 \\
M \\
23\end{array}$ & C6-7 & 4.0 & $+++(\mathrm{Bil})$ & Yes & 0.40 & 0.40 & 2 canes \\
\hline $\begin{array}{r}5 \\
\mathrm{M} \\
32\end{array}$ & C7-T1 & 1.5 & $+++($ Bil $)$ & Yes & 0.15 & 0.25 & Walker \\
\hline $\begin{array}{l}7 \\
M \\
20\end{array}$ & Т8-9 & 1.5 & & Yes & 0.15 & 0.15 & 2 canes \\
\hline $\begin{array}{r}8 \\
M \\
42\end{array}$ & T9-10 & 0.6 & $+++(\mathrm{Bil})$ & $(\mathrm{L}>\mathrm{R})$ & 0.10 & 0.15 & $\begin{array}{l}(\mathrm{L}) \text { foot drop } \\
\text { brace; walker }\end{array}$ \\
\hline
\end{tabular}


8) lower limb while the subjects walked on the treadmill. When subjects presented with asymmetrical involvement of the lower extremities (i.e. one limb showing minimal spasticity and weakness), data were collected from the more involved lower limb. When the degree of involvement of the lower limbs was largely symmetrical, data were then collected from the limb that showed a greater degree of clinical spasticity. Bipolar surface electrodes $(2.5 \mathrm{~cm}$ center to center) were placed over the belly of each muscle following conventional skin preparation. The EMG signals were preamplified, differentially amplified and bandpassed $(10-470 \mathrm{~Hz})$. Footswitches placed under the heel, fifth metatarsal head and big toe of each subject's shoes were used to detect heel strike, foot-flat and toe-off, and to determine the temporal distance parameters. The EMG and footswitch signals were then recorded at 3.75 IPS on a 14 channel FM tape with a frequency response of $2500 \mathrm{~Hz}$.

The EMG signals of the six muscles along with the footswitch signals were then played back on a polygraph and an artefact-free sequence of 10 or more consecutive cycles was chosen to represent each experimental paradigm for each subject.

\section{Joint angular displacement data}

Joint angular displacement data were collected from the same limb as the EMG recordings for each subject. The subjects were videotaped as they walked on the treadmill using a shutter video camera. Reflective joint markers were placed at the shoulder, hip, knee and ankle as well the heel, fifth metatarsal head and the toe region of the lateral border of the shoe. Additional markers were placed on a vertical and a horizontal bar to be used as absolute coordinates for the video analysis. The trials were recorded on a $3 / 4$ inch videotape at a speed of 60 fields per second. A remote search controller was used for field by field viewing. The sagittal angular displacements were manually measured from the monitor screen using a goniometer. Once the subjects had reached a steady state while walking on the treadmill, one representative gait cycle for each subject during each experimental paradigm was analyzed. The joint angular displacements were measured at every $5 \%$ of the gait cycle. The trunk and hip angles were calculated with respect to a vertical line, with the neutral position in standing being taken as displacement of the trunk and hip, flexion being positive, and extension negative. Likewise, in calculating the knee and ankle angles, the neutral standing position, with the knee in full extension and the shank axis perpendicular to the foot, was taken as $0^{\circ}$. Knee flexion and ankle dorsiflexion beyond neutral were taken as positive angular displacements, and ankle plantarflexion beyond neutral was taken as negative angular displacement.

\section{Results}

\section{Effects of parallel bars and BWS}

The effects of removing parallel bar support during treadmill locomotion at comfortable speeds, at $0 \%$ and $40 \%$ BWS will be addressed both in those subjects with an asymmetrical and a symmetrical gait pattern.

Figure 1 illustrates the EMG patterns of subject 2 who walked with an asymmetrical gait pattern due to marked impairment of the right lower limb and a near normal left lower limb. He walked on a treadmill with (Figure 1a) and without parallel bars (Figure 1b) at $0 \%$ BWS and without parallel bars at $40 \%$ BWS (Figure 1c) at his comfortable speed of $0.08 \mathrm{~ms}^{-1}$. While walking with parallel bars (Figure 1a), he compensated for his lack of hip, knee and ankle flexion during the right swing phase by pushing on the parallel bars to come up onto his left toes while swinging the right limb through fully extended. As illustrated in Figures $1 \mathrm{e}-\mathrm{g}$, there was no flexion at the hip or ankle and minimal flexion at the knee $\left(11^{\circ}\right)$ during the right swing phase. The EMG profiles (Figure 1a) revealed activity in most right lower limb muscles during the stance phase. Low level activity appeared in TA during stance with minimal activity during the swing phase. During the same experimental session, the subject was asked to walk without parallel bars (Figure 1b). As 

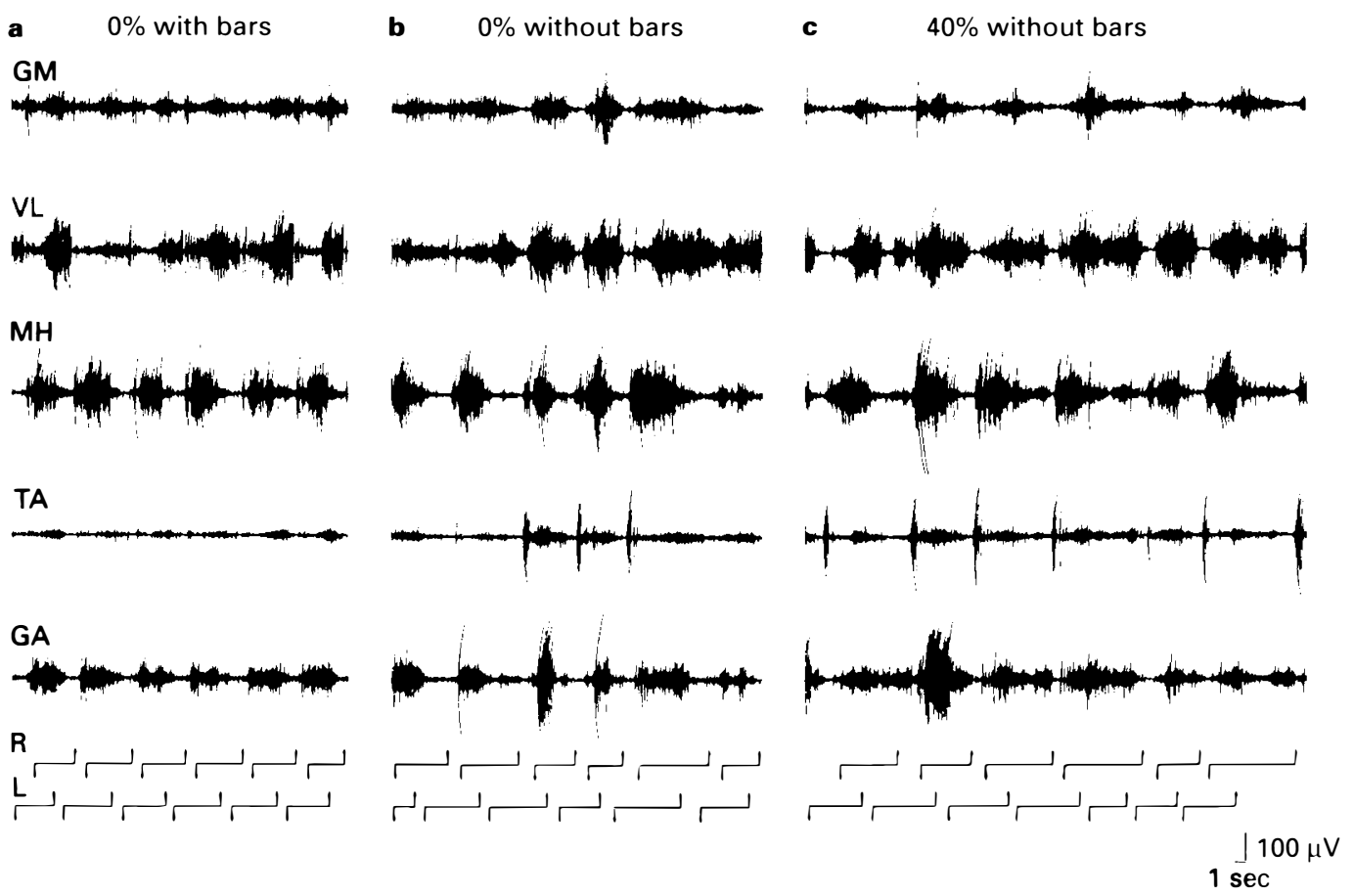

Figure 1a-c Caption on p. 545.

he released the parallel bars, his legs dragged at the end of the treadmill following which he was able to initiate and complete only three laborious steps (Figure 1b). During these three steps a marked increase in hip and knee flexion as well as ankle dorsiflexion emerged as the subject was no longer able to compensate by using the parallel bars, but required flexion of the right lower extremity in order to advance the limb forward (Figure $1 \mathrm{~d}-\mathrm{g}$ ). The most evident EMG change was seen in TA during the swing phase where a burst of activity appeared resulting in ankle dorsiflexion. Although walking without parallel bars was very difficult for this subject, it facilitated a more normal swing phase, with flexion at the hip, knee and ankle.

When $40 \%$ BWS was provided (Figure 1c) a much smoother, less strenuous gait resulted, thereby allowing the subject to take a greater number of steps (a minimum of 10 steps per trial). TA continued to show a burst of activity during the swing phase. The sagittal angular displacement profiles (Fig 1d-g) revealed a considerably straighter knee at foot-floor contact $\left(30^{\circ}\right)$ when compared to $0 \%$ BWS $\left(56^{\circ}\right)$. Ankle dorsiflexion was initiated earlier in late stance.

Similar findings were observed in subject 3 (not illustrated) who also walked with an asymmetrical gait pattern by compensating with her less involved right side in a similar way to subject 2 . When the subject walked without parallel bars at $0 \% \mathrm{BWS}$, a burst of activity appeared in TA during the swing phase which was not present when the parallel bars were used. This corresponded to an increase in ankle dorsiflexion during the swing phase, similar to that seen in subject 2 .

Facilitation of gait with $40 \%$ BWS was also seen in subject 3 when BWS resulted in a smoother, less spastic gait.

Figure $2 \mathrm{a}-\mathrm{c}$ illustrates the effects of walking with and without parallel bars at $0 \%$ BWS and without parallel bars at $40 \%$ BWS in a symmetrical subject (6) at $0.43 \mathrm{~ms}^{-1}$. Except for prolonged VL activation (Fig 2a), this subject's lower limb EMG patterns were similar to those seen in 

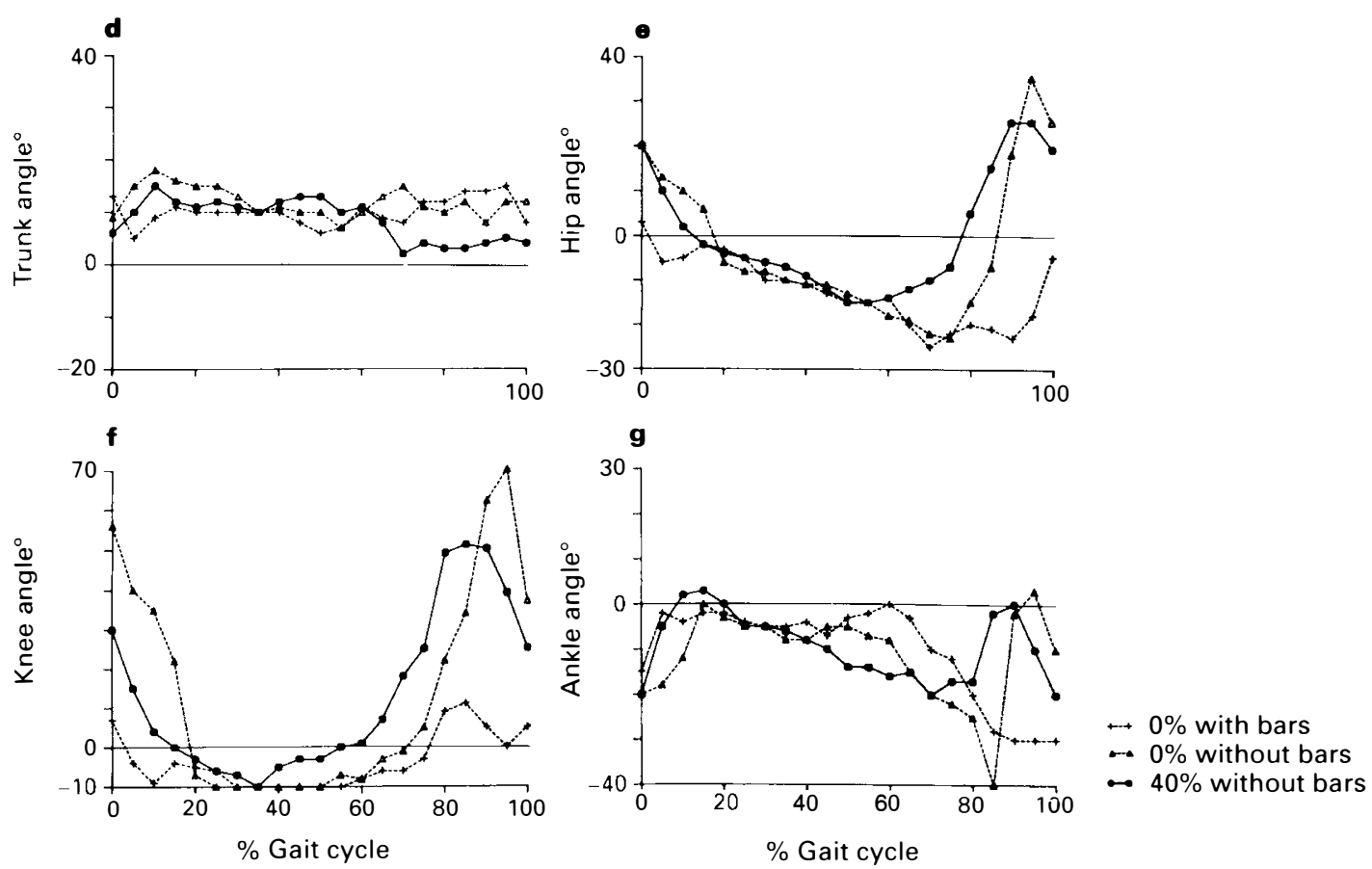

Figure 1 The right lower limb EMG activity of subject 2 walking on the treadmill, at a speed of $0.08 \mathrm{~ms}^{-1}$, at (a) $0 \%$ BWS with parallel bars, (b) $0 \%$ BWS without parallel bars, and (c) $40 \%$ BWS without parallel bars. The downward arrows indicate foot-floor contact, while the upward arrows indicate toe-off, with the solid line depicting stance duration and the space denoting swing duration, for both right (R) and left (L) lower limbs. In (b), the second, third and fourth steps of the right lower limb represent the three steps the subject takes without parallel bars. In (b) and (c), note the burst of activity in TA during the swing phase. In (c), note that the subject is able to take a greater number of steps without parallel bars when $40 \%$ BWS is provided. The corresponding sagittal angular displacement patterns of a representative cycle for the (d) trunk, (e) hip, (f) knee and (g) ankle are also illustrated. Note the increase in hip and knee flexion and ankle dorsiflexion during the swing phase when walking without parallel bars and the straighter knee at foot-floor contact when $40 \%$ BWS is provided.

normal subjects. ${ }^{8,9}$ When the subject walked without parallel bars (Fig 2b), an increase in EMG activity of all lower extremity muscles was noted especially at foot-floor contact. TA showed an increase in tonic activity during stance. A broadening of both the GA and SOL bursts were noted with early activation at foot-floor contact. This was accompanied by a large increase in EMG amplitude especially in the GA muscle. Except for a small decrease in plantarflexion at the ankle at the end of stance, walking without parallel bars resulted in minimal changes in lower limb angular displacement profiles in this subject (Fig $2 \mathrm{~d}-\mathrm{g}$ ). Similar findings were noted in subject 1 (not illustrated).
In subject 6 , providing $40 \%$ BWS during treadmill locomotion without parallel bars resulted mainly in a decrease in EMG amplitudes for all lower extremity muscles. This is exemplified in Figure 2c where the EMG profiles closely resembled those at $0 \%$ BWS with parallel bars, illustrated in Figure 2a. Minimal changes were noted in the sagittal angular displacement patterns when contrasting $0 \%$ and $40 \%$ BWS in this subject (Fig 2d-g). A similar trend was noted in subject BP at his minimal treadmill speed of $0.20 \mathrm{~m}^{-1}$.

Providing $40 \%$ BWS also facilitated gait without parallel bars in two more severely impaired subjects $(5,7)$ with a symmetrical gait pattern. These subjects were not able to 


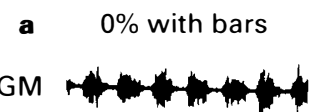

VL

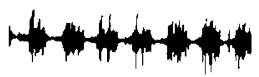

$\mathrm{MH}$

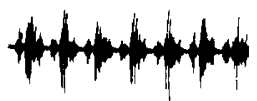

TA

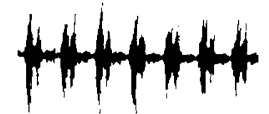

GA

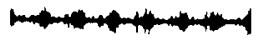

SOL

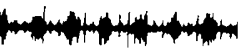

L

R

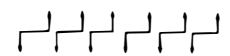

r
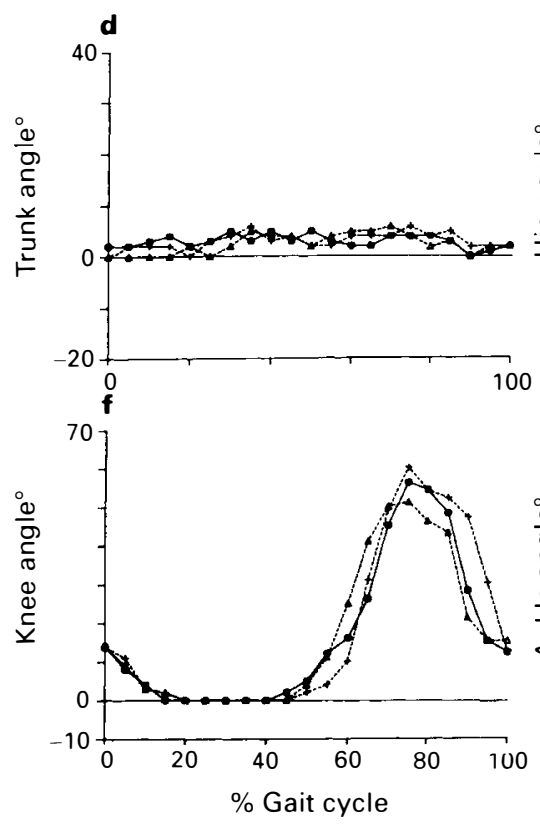

b $0 \%$ without bars
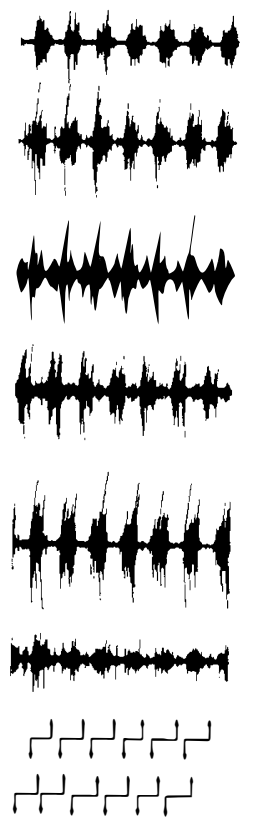

c $40 \%$ without bars
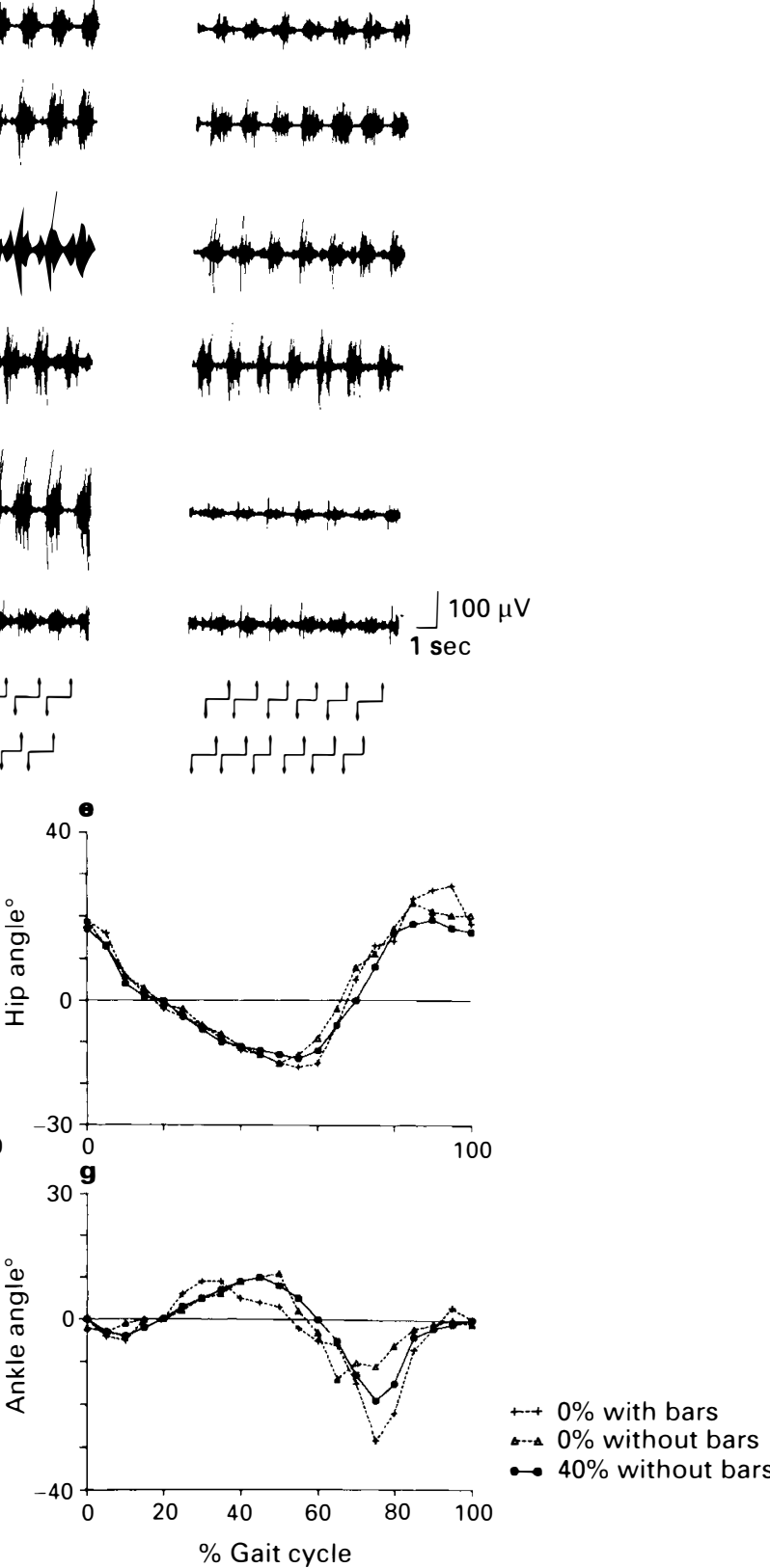

Figure 2 The left limb EMG activity of subject 6 walking on the treadmill, at a speed of $0.43 \mathrm{~ms}^{-1}$, at (a) $0 \%$ BWS with parallel bars, (b) $0 \%$ BWS without parallel bars, and (c) $40 \%$ BWS without parallel bars. The downward arrows indicate foot-floor contact, while the upward arrows indicate toe-off, with the solid line depicting stance duration and the space denoting swing duration, for both left (L) and right (R) lower limbs. In (b), note the increase in VL activity as well as the broadening of activity in TA, GA and SOL during stance. The corresponding sagittal angular displacement patterns of a representative cycle for the (d) trunk, (e) hip, (f) knee and (g) ankle are also illustrated. 
walk without parallel bars at $0 \%$ BWS but were able to do so when $40 \%$ BWS was provided.

\section{$B W S$ versus parallel bars}

In those subjects $(2,3)$ who walked with an asymmetrical, compensatory gait pattern, $40 \%$ BWS appeared to have the most positive effects on the gait parameters when the subjects walked without parallel bars. This is illustrated in Figure $3 \mathrm{a}-\mathrm{g}$ which shows the effects of $40 \%$ BWS with and without parallel bars. As subject 2 walked on the treadmill with parallel bars at $0 \%$ BWS he compensated by supporting himself on the parallel bars, coming up onto his left toes during left stance, while swinging the right leg forwards with a minimal amount of hip, knee and ankle flexion. When $40 \%$ BWS was provided, while he walked with parallel bars, minimal changes were noted in the gait pattern (Figure $3 \mathrm{~b}, \mathrm{~d}-\mathrm{g}$ ) as the subject continued to compensate. However, when the subject released the parallel bars while walking at $40 \%$ BWS, a more normal gait pattern emerged. This was characterized by an appropriate swing phase with flexion at the hip, knee and ankle (Fig 3c-g) and a burst of activity in TA (Fig 3c).

\section{Effects of speed}

Increasing treadmill speed, at $0 \%$ BWS while walking with parallel bars, resulted in an increase in clonus of the distal muscles for subjects 3, 7 and 8 . In subject 1 , clonus was not present at the slowest speed, but was elicited at the higher speeds. An example is illustrated in Figure $4 \mathrm{a}-\mathrm{g}$ for subject 8 with increases in treadmill speed from $0.05 \mathrm{~ms}^{-1}$ to $0.15 \mathrm{~ms}^{-1}$. At the lowest speed of $0.05 \mathrm{~ms}^{-1}$ (Fig 4a), coactivation of the extensor muscles was noted. There was persistent activity in GM and VL throughout stance. Minimal activity was evident in MH. A burst of activity during the swing phase was noted in TA. GA was characterized by low tonic activity during stance with

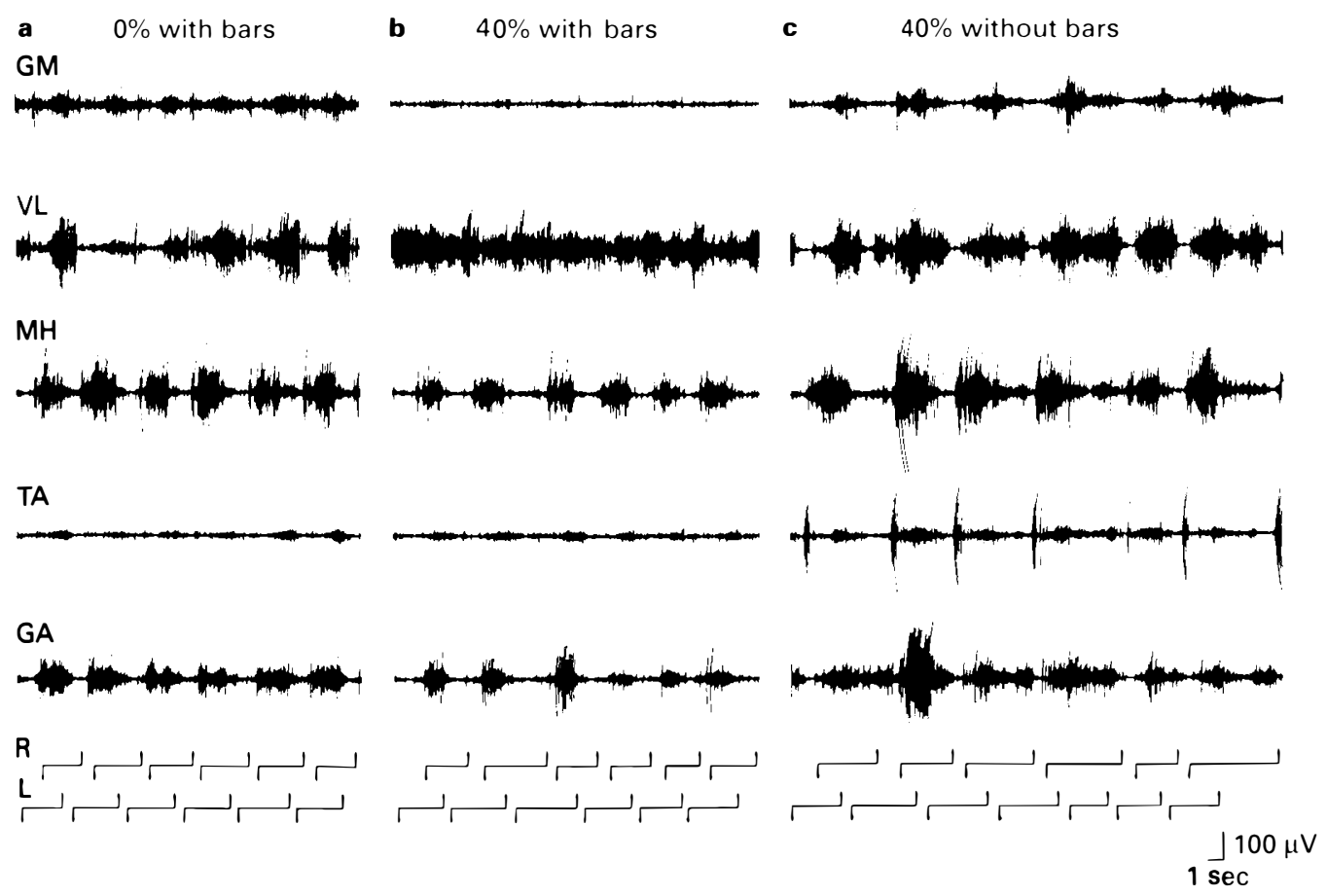

Figure 3a-c Caption on p. 548. 

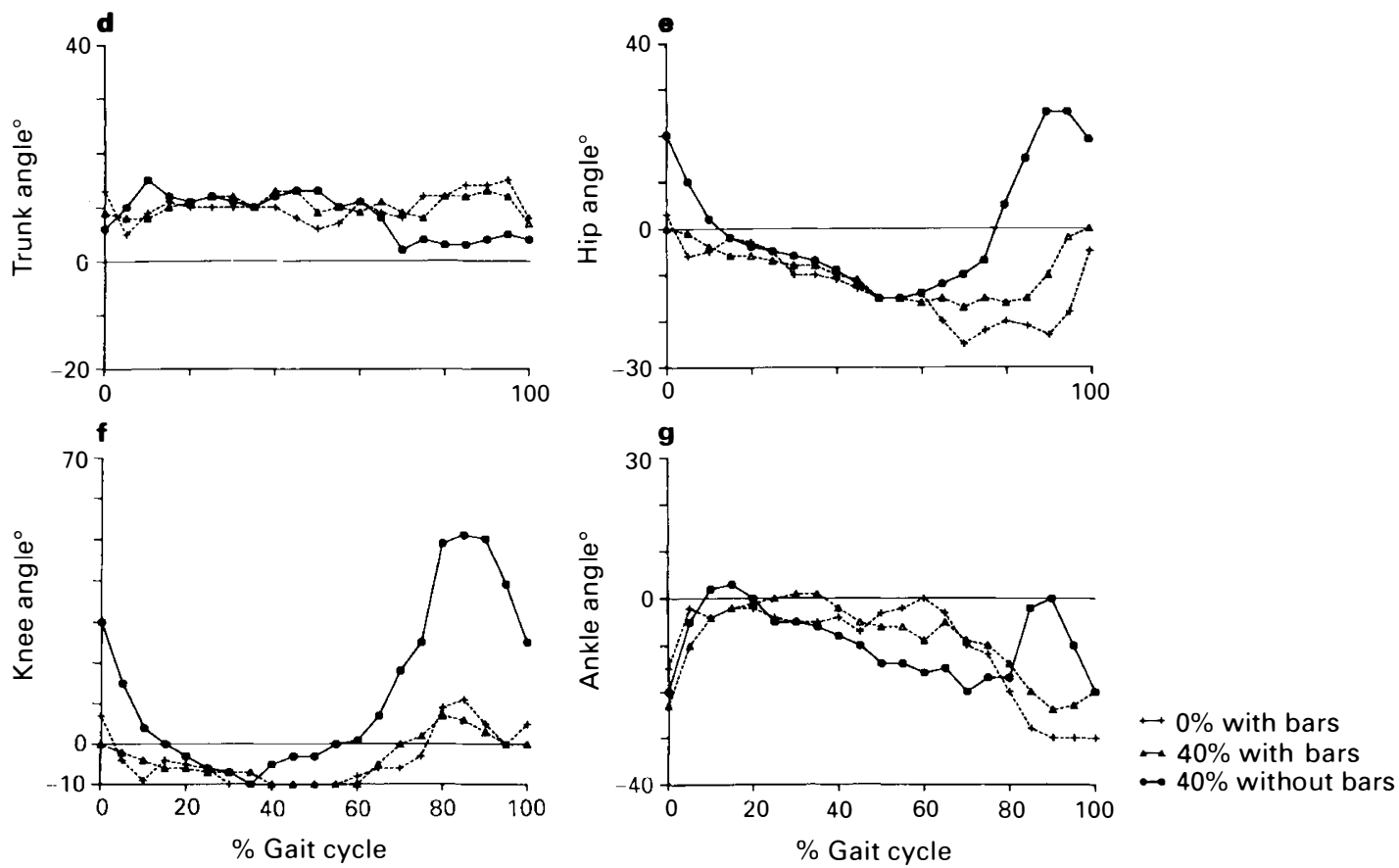

Figure 3 The right lower limb EMG activity of subject 2 walking on the treadmill, at a speed of $0.08 \mathrm{~ms}^{-1}$, at (a) $0 \%$ BWS with parallel bars, (b) $40 \%$ BWS with parallel bars, and (c) $40 \%$ BWS without parallel bars. The downward arrows indicate foot-floor contact, while the upward arrows indicate toe-off, with the solid line depicting stance duration and the space denoting swing duration, for both right $(\mathrm{R})$ and left $(\mathrm{L})$ lower limbs. In (c), note the more phasic EMG activity in VL and the appearance of a burst of activity in TA during swing. The corresponding sagittal angular displacement patterns of a representative cycle for the (d) trunk, (e) hip, (f) knee and (g) ankle are also illustrated. Note the presence of hip and knee flexion and ankle dorsiflexion at $40 \%$ BWS without parallel bars.

the onset of low amplitude clonus shortly after initial foot-floor contact. SOL also showed clonus during that period, followed by a burst of activity continuing until terminal stance. Increasing the treadmill speed to $0.10 \mathrm{~ms}^{-1}$ and $0.15 \mathrm{~ms}^{-1}$ (Fig $4 \mathrm{~b}, \mathrm{c}$ ) resulted in sustained clonus of all muscles during the entire stance phase and, in some instances, during early swing. This resulted in a laboured gait, especially at the highest speed, characterized by increased difficulty walking and clonic oscillations visible at the ankle.

Figure $4 \mathrm{~d}$ revealed a straighter trunk alignment at the higher speeds for subject 8 . Minimal changes in the hip angular excursion patterns were evident (Fig 4e). At the higher speeds an increase in knee flexion during mid to terminal stance was noted, with a small decrease in maximum swing angle (Fig 4f). A change in the ankle kinematic profiles was the occurrence of plantarflexion during midstance as the subject went up on his toes to facilitate swinging the left leg through (Fig 4g). An increase in plantarflexion at push-off was also noted.

In subjects 4,5 and 6 who showed minimal abnormal reflex activity in the distal muscles during locomotion, increases in speed had a minimal effect on EMG timing and amplitude (not illustrated). The effects were to produce a small increase in EMG amplitude for lower extremity muscles such as the GM, TA, GA and SOL. Qualitatively, the gait appeared more laboured at the higher speeds. 
a

GM

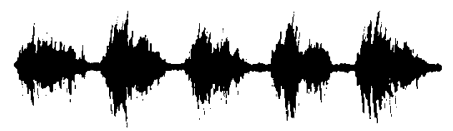

VL

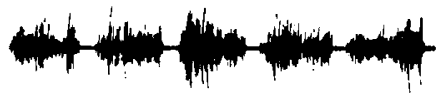

$\mathrm{MH}$

TA

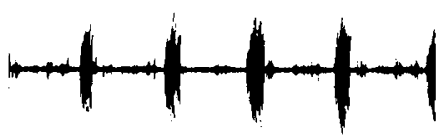

GA

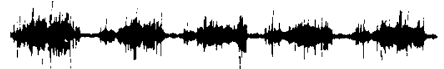

SOL

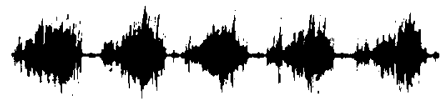

$\mathrm{R}$

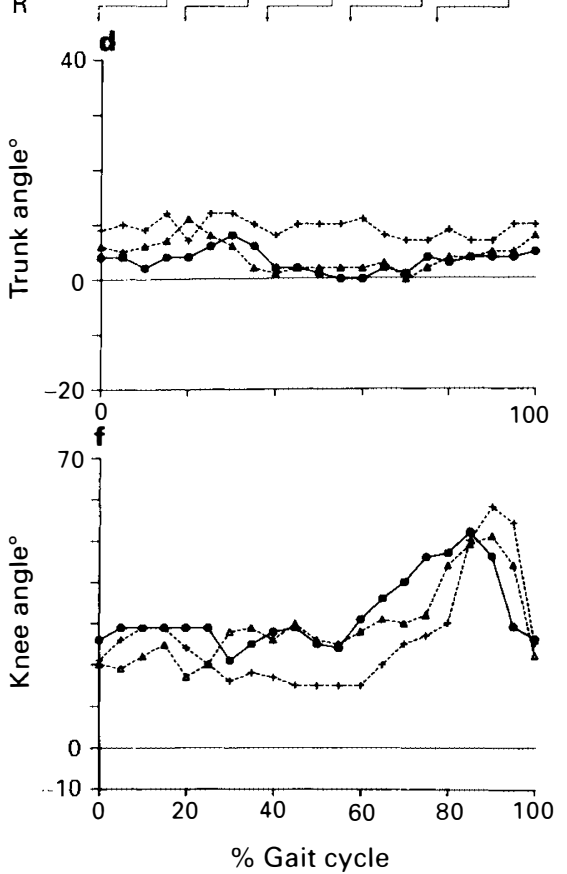

b
$0.10 \mathrm{~ms}$
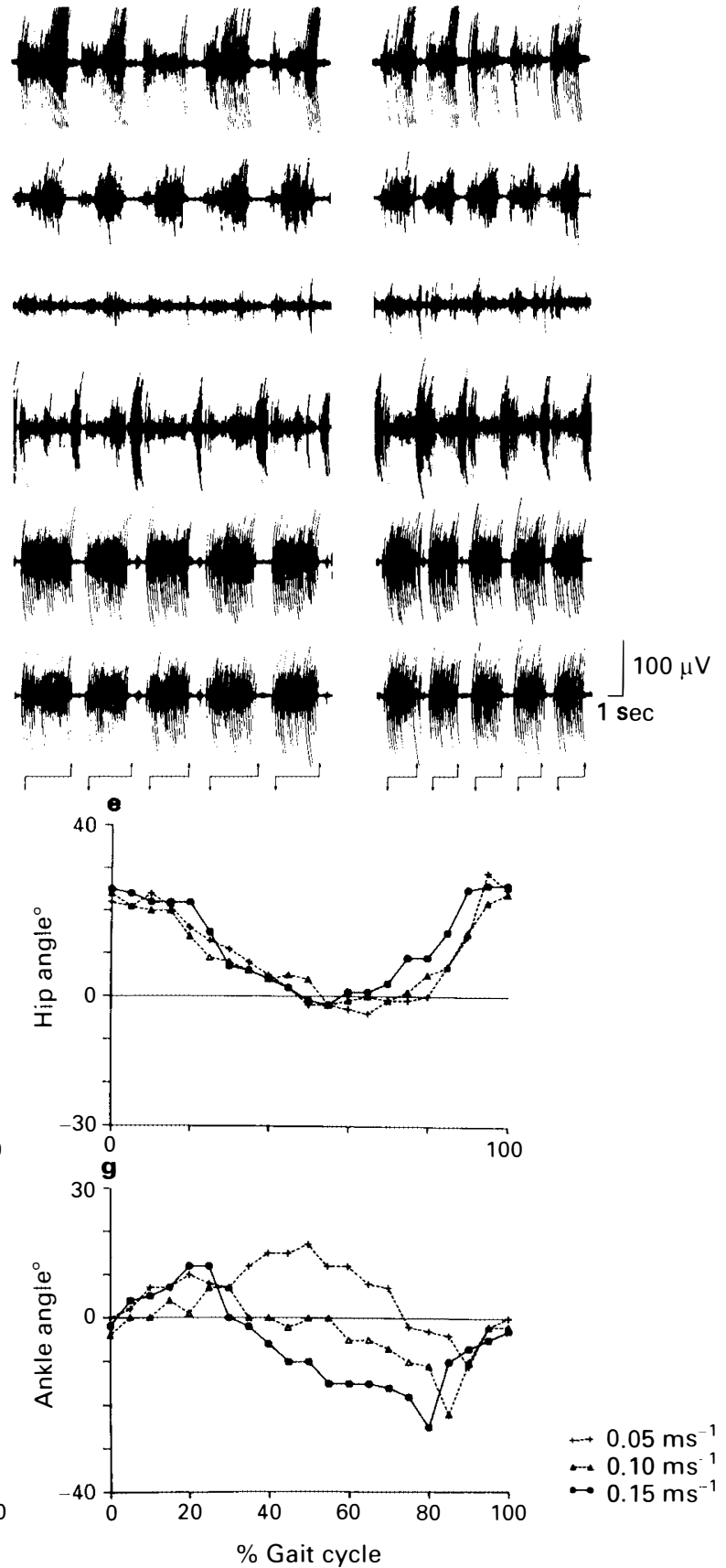

c $\quad 0.15 \mathrm{~ms}^{1}$
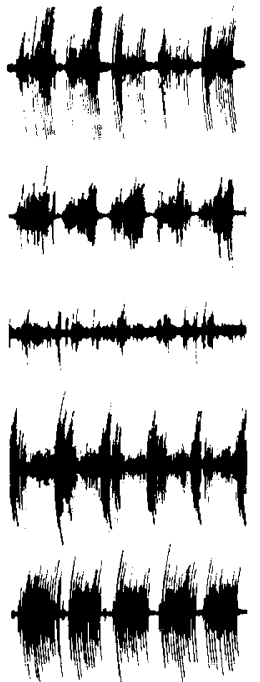

Figure 4 The right lower limb EMG activity of subject 8 walking on the treadmill, with parallel bars, at (a) $0.05 \mathrm{~ms}^{-1}$, (b) $0.10 \mathrm{~ms}^{-1}$, and (c) $0.15 \mathrm{~ms}^{-1}$. The downward arrows indicate foot-floor contact, while the upward arrows indicate toe-off, with the solid line depicting stance duration and the space denoting swing duration, for the right $(\mathrm{R})$ lower limb. Note the presence of sustained clonus at the higher treadmill speeds. The corresponding sagittal angular displacement patterns of a representative cycle for the (d) trunk, (e) hip, (f) knee and (g) ankle are also illustrated. 


\section{Discussion}

\section{Effects of parallel bars and body weight support}

Ambulating without parallel bars has been deemed by some investigators to accentuate gait disturbances in spastic syndromes. ${ }^{1,3,10}$ In this study, removing parallel bars in symmetrically involved spastic paretic subjects resulted in an increase and prolongation of EMG activity during stance. Conrad et $a l^{3}$ attributed comparable EMG changes, in a group of spastic paretic subjects, to nonspecific protective gait mechanisms present during instances of emotional stress or anxiety when the subject's stability is threatened. However, in the present experiment, subjects were securely supported in the harness even when no BWS was provided (under full weight bearing conditions) while they walked without parallel bars. All subjects reported that the harness provided a feeling of safety which eliminated any fear of falling. Regardless of this, a deterioration in the gait pattern characterized by an increase and broadening of the EMG activity was noted in subjects with symmetrical gait involvement. These results suggest that parallel bars provide lateral stability which compensates for the decreased postural reactions observed in spastic subjects ${ }^{10}$ while walking on a moving surface. The changes in EMG patterns especially noted in the distal muscles, closely resemble those observed in an immature gait when postural reactions are not yet fully developed. ${ }^{11-13}$ Providing 40\% BWS for such patients resulted in more normal EMG profiles and it can be suggested that BWS compensated for the decrease in postural stability. This is supported by a previous study which reports that providing $40 \%$ BWS, while walking on a treadmill, results in a decrease in percentage total double support time and an increase in single limb support time in spinal cord injured subjects.

An interesting observation was the more symmetrical gait pattern which emerged in asymmetrically involved subjects when they walked without holding on to parallel bars at $0 \%$ BWS. Although the gait was laborious and nonfunctional (limited to three steps), removing the parallel bars produced a more normal swing phase by eliciting EMG activity in TA accompanied by ankle dorsiflexion. The hip, knee, and ankle flexion present when walking without parallel bars had not emerged under conditions where the subject was allowed to compensate by using parallel bars. The more normal gait pattern only emerged with an increase in locomotor demand and no possibility for compensation.

In order to facilitate gait when asymmetrical subjects walked without parallel bars, $40 \%$ BWS was provided. The subjects were able to take a larger number of steps with greater ease, making gait training without parallel bars more feasible. Severely impaired subjects with symmetrical involvement, who were not able to walk without parallel bars at $0 \% \mathrm{BWS}$, were able to do so when $40 \%$ BWS was provided. Retraining gait with BWS, while increasing the demand on the locomotor system by removing parallel bars, is worth considering in the early stages of gait training. The approach appears imperative in facilitating a more symmetrical gait pattern while discouraging gait asymmetries from developing. Although both parallel bars and BWS can be considered as a form of support during gait training, there appears to be definite differences between the two. Parallel bars appear to yield a more compensatory gait pattern, probably by facilitating weight transference to the less involved side in asymmetrically involved subjects. BWS encourages a more symmetrical gait pattern by supporting a percentage of body weight centrally, and discouraging compensation with the less involved extremity. The differences between these two types of support for both symmetrical and asymmetrical patients need to be further elucidated.

\section{The effects of increasing treadmill speed}

Variations in EMG activity, angular displacement profiles, and temporal distance parameters as a function of walking speed in healthy subjects have been extensively reported in the literature ${ }^{14-17}$. This is in contrast to a lack of objective information which exists quantifying the effects of in- 
creased walking speed on gait parameters in spinal cord injured patients. Diet ${ }^{18}$ alluded to muscle hypertonia as one of the main reasons for the spastic paretic subjects' inability to walk at faster speeds.

In this study, one of the effects of increasing walking speed was the appearance or increase in clonus in four subjects. Burke \& Lance $^{19}$ have reported that the amplitude of stretch reflexes are directly proportional to the velocity of stretch. In three other subjects, increasing walking speed resulted in only a small increase in muscle EMG amplitude.

Spastic paretic subjects are known to walk at speeds considerably lower than that of normal subjects. ${ }^{5,6}$ In the present group of subjects, the comfortable treadmill speeds ranged from $0.08 \mathrm{~ms}^{-1}$ to $0.40 \mathrm{~ms}^{-1}$, and the highest maximal treadmill speed was $0.60 \mathrm{~ms}^{-1}$ for subject 1 . At such low walking speeds it becomes imperative to distinguish gait characteristics caused by the pathology and those which are a result of slow walking speeds. ${ }^{20.21}$ Until recently, ${ }^{22}$ pathological gait profiles were compared to standardized normative data derived from healthy subjects walking at comfortable speeds. . $^{1.23}$ Shiavi et al $^{16}$ investigated the EMG profiles of healthy subjects walking at very low speeds (lowest range: $0.34 \mathrm{~ms}^{-1}$ ) in order to provide a template for comparison with pathological gait while controlling the speed effect. They reported that $\mathrm{MH}$ muscle was biphasic at the very low walking speeds, with most subjects displaying a burst of activity at the stance-swing transition which was abolished as the speed increased. This type of MH EMG profile has been observed in this as well as other studies ${ }^{1,5}$ and may be a result of low walking speed and not necessarily of abnormal motor programming. Shiavi et al ${ }^{16}$ also reported that gait parameters became more variable at the very low walking speeds and that muscles responded to individual movement requirements. Subjects described their gait as becoming less automatic. The low walking speeds may partially explain the increased variability in gait parameters observed among spastic paretic subjects when compared to normal subjects. ${ }^{24}$ Friso et al ${ }^{14}$ studied lower limb angular displacements patterns in normal subjects at different walking speeds and reported the frequent absence of a yield at the knee during initial stance at lower speeds. This is a finding frequently observed in spastic paretic gait, ${ }^{5}$ and the subjects' low walking speeds may be partly responsible for this.

In general, subjects in this study exhibited a low EMG amplitude for most muscles while walking at their comfortable treadmill speeds. Knutsson ${ }^{24}$ described one of the abnormal activation patterns as a marked decrease in EMG activity during gait in certain muscles, although the subjects were able to generate force in these muscles during other tasks. The decreases in EMG activity noted among spastic paretic subjects may partly be resulting from their low walking speeds as well as central paresis.

\section{Implications for gait training}

The complexity of the disturbed locomotor pattern, coupled with postural instability and abnormal reflex activity, following a spinal cord lesion, make it difficult to establish a universal training strategy to reeducate gait. A clear understanding of the gait deficits and their causes is required in individual cases in order to propose a comprehensive gait training approach. ${ }^{10,24,25}$ Likewise, an understanding of the influence of external parameters such as parallel bars, BWS and speed are needed in order to incorporate them into a training strategy. Although removal of parallel bars has been thought to produce a deterioration in the gait pattern, it has been demonstrated here that in patients with an asymmetrical gait pattern, removing parallel bars will decrease the opportunity for compensation thus allowing for the expression of a more normal gait pattern. If without parallel bars the locomotor task is a difficult one, BWS can be incorporated in the training regimen to facilitate gait. For those subjects who are unable to walk without parallel bars at $0 \%$ BWS, providing $40 \%$ BWS allows gait training without parallel bars and minimizes the development of a compensatory gait pattern. In symmetrically involved patients parallel bars can be removed and postural stability increased with BWS while retrain- 
ing gait. Such patients appear to be candidates for gait training with BWS, achieving the goal of increasing speed, increasing postural stability, while eliciting more normal EMG and angular displacement profiles of the lower extremities.

Increasing walking speed did not always result in a deterioration in the gait pattern of spastic paretic subjects. Those presenting with minimal abnormal reflex activity during gait showed just a small increase in EMG amplitude although they were not able to walk at speeds much beyond that of their comfortable level (subject 5: $0.15 \mathrm{~ms}^{-1}-0.25 \mathrm{~ms}^{-1}$; subject $1: 0.40 \mathrm{~ms}^{-1}$ $\left.0.60 \mathrm{~ms}^{-1}\right)$. Such subjects would probably benefit from gait training with BWS at speeds higher than their comfortable walking speed. ${ }^{5}$ In subjects where increased speed led to an increase in abnormal reflex activity, drug therapy would be indicated prior to gait training. ${ }^{6,26,27}$

When retraining gait and other functional tasks, it becomes important to manipulate the environment and provide external conditions which favour the response being sought and enhance functional recovery. ${ }^{28}$ Providing the appropriate environmental conditions is critical during gait training in order to maximize the locomotor potential while preventing compensatory gait deviations. It becomes clear from the above results that following a spinal cord injury a comprehensive and interactive approach is required in order to maximize the potential for locomotor recovery. An understanding of the underlying abnormal motor programs and reflex activity, coupled with an understanding of the influence of external parameters such as parallel bars, BWS and speed during locomotion, are essential to achieve this goal.

\section{References}

1 Conrad B, Benecke R, Meinck HM (1985) Gait disturbances in paraspastic patients. In: Delwaide PJ, Young RR, editors. Clinical Neurophysiology in Spasticity. Vol 1. Restorative Neurology. Elsevier Science Publishers B.V. (Biomedical Division), Netherlands: 155-174.

2 Knutsson E (1980) Muscle activation patterns of gait in spastic hemiparesis, paraparesis and cerebral palsy. In: Fugel-Meyer A, editor. Stroke with Hemiplegia. Scand J Rehabil (Suppl 7): 47-52.

3 Conrad B, Benecke R, Carnehl J et al (1983) Pathophysiological aspects of human locomotion. In Desmedt JE, editor. Motor Control Mechanisms in Health and Disease. Raven Press, New York: 717-726.

4 Bobath B (1978) Adult Hemiplegia: Evaluation and Treatment. 2nd edn. Heinnemann Medical Books, London, UK.

5 Visintin M, Barbeau H (1989) The effects of body weight support on the locomotor pattern of spastic paretic patients. Can J Neurol Sci 16: $315-325$.

6 Barbeau H, Fung J, Stewart J, Visintin M (1988) Impairment of spastic paraparetic gait: implications for new rehabilitation strategies. Proc Fifth Biennial Conf Can Soc Biomech: 12-16.

7 Barbeau H, Wainberg M, Finch L (1987) Description and application of a system for rehabilitation. Med Bio Eng Comput 25: 341-344.

8 Fung J, Barbeau H (1987) Quantification of the electromyographic activity in normal human gait. Proc IEEE Conf Biomed Technol, Montech: 41-44.

9 Hirschberg GG, Nathanson M (1952) Electromyographic recording of muscular activity in normal and spastic gaits. Arch Phys Med Rehabil 33: 217-226.

10 Benecke R, Conrad B (1986) Disturbances of posture and gait in spastic syndromes. In: Bles W, Brandt TH, editors. Disorders of Posture and Gait. Elsevier Science Publishers B.V. (Biomedical Division), Netherlands: 231-241.

11 Berger W (1986) Development of gait in children. In: Bles W, Brandt TH, editors. Disorders of Posture and Gait. Elsevier Science Publishers B.V. (Biomedical Division): 315-324.

12 Forssberg H (1985) Ontogeny of human locomotor control I. Infant stepping, supported locomotion and transition to independent locomotion. Exp Brain Res 57: 480-493.

13 Sutherland DH, Olshen R, Cooper LB, Woo SLY (1980) The development of mature gait. J Bone Joint Surg Am 62: 336-353.

14 Frigo C, Eng D, Tesio L (1986) Speed-dependant variations of lower-limb joint angles during walking. Am J Phys Med 65: 51-62.

15 Kirtley C, Whittle MW, Jefferson RJ (1985) Influence of walking speed on gait parameters. J Biomed Eng 7: 282-288.

16 Shiavi R, Bugle HJ, Limbird T (1987) Electromyographic gait assessment, part 1: Adult EMG profiles and walking speed. J Rehabil Res Dev 24: 13-23. 
17 Yang JF, Winter DA (1985) Surface EMG profiles during different walking cadences in humans. EEG Clin Neurophysiol 60: $485-491$.

18 Dietz V (1986) Impaired reflex control of posture and gait in spastic paresis. In: Bles W, Brandt $\mathrm{TH}$, editors. Disorders of Posture and Gait. Elsevier Science Publishers B.V. (Biomedical Division), Netherlands: 243-252.

19 Burke DC, Lance JW (1973) Studies of the reflex effects of primary and secondary spindle endings in spasticity. In: Desmedt JE, editor. New Developments in Electromyography and Clinical Neurophysiology. Vol 3. Karger, Basal: 475-495.

20 Andriacchi TP, Ogle JA, Galante JO (1977) Walking speed as a basis for normal and abnormal gait measurements. J Biomech 10: 261-264.

21 Longhurst S (1980) Variability of EMG during slow walking. Proc Special Conf Can Soc Biomechan: $10-11$.

22 Shiavi R, Bugle HJ, Limbird T (1987) Electromyographic gait assessment, part 2: Preliminary assessment of hemiparetic synergy patterns. J Rehabil Res Dev 24: 24-30.

23 Knutsson E, Richards C (1979) Different types of disturbed motor control in gait of hemiparetic patients. Brain 102: 405-439.

24 Knutsson E (1985) Studies of gait control in patients with spastic paresis. In: Delwaide PJ, Young RR, editors. Clinical Neurophysiology in Spasticity. Vol 1. Restorative Neurology. Elsevier Science Publishers B.V. (Biomedical Division), Netherlands: 175-183.

25 Grimm RJ (1983) Program disorders of movement. In: Desmedt JE, editor. Motor Control Mechanisms in Health and Disease. Raven Press, New York: 1-11.

26 Barbeau H, Richards CL, Bedard PJ (1982) Action of cyproheptadine in spastic paraparetic patients. J Neurol Neurosurg Psychiatry 45: 923-926.

27 Wainberg M, Barbeau H, Gauthier S (1986) Quantitative assessment of the effects of cyproheptadine on spastic paretic gait: A prelimianry report. J Neurol 233: 311-314.

28 Bach-y-Rita P (1983) Rehabilitation versus passive recovery of motor control following central nervous system lesions. In: Desmedt JE, editor. Motor Control Mechanisms in Health and Disease. Raven Press, New York: 1085-1092. 